\title{
AAPS Workshop Report: Strategies to Address Therapeutic Protein-Drug Interactions during Clinical Development
}

\author{
Sandhya Girish, ${ }^{1,11}$ Steven W Martin, ${ }^{2}$ Mark C Peterson, ${ }^{3}$ Lei K. Zhang, ${ }_{7}^{4}$ Hong Zhao, ${ }^{4}$ Joseph Balthasar, \\ Raymond Evers, ${ }^{6}$ Honghui Zhou, ${ }^{7}$ Min Zhu, ${ }^{8}$ Lewis Klunk, ${ }^{9}$ Chao Han, ${ }^{7}$ Eva Gil Berglund, ${ }^{10}$ \\ Shiew-Mei Huang, ${ }^{4}$ and Amita Joshi ${ }^{1}$
}

Received 16 February 2011; accepted 27 April 2011; published online 1 June 2011

\begin{abstract}
Therapeutic proteins (TPs) are increasingly combined with small molecules and/or with other TPs. However preclinical tools and in vitro test systems for assessing drug interaction potential of TPs such as monoclonal antibodies, cytokines and cytokine modulators are limited. Published data suggests that clinically relevant TP-drug interactions (TP-DI) are likely from overlap in mechanisms of action, alteration in target and/or drug-disease interaction. Clinical drug interaction studies are not routinely conducted for TPs because of the logistical constraints in study design to address pharmacokinetic (PK)and pharmacodynamic (PD)-based interactions. Different pharmaceutical companies have developed their respective question- and/or risk-based approaches for TP-DI based on the TP mechanism of action as well as patient population. During the workshop both company strategies and regulatory perspectives were discussed in depth using case studies; knowledge gaps and best practices were subsequently identified and discussed. Understanding the functional role of target, target expression and their downstream consequences were identified as important for assessing the potential for a TP-DI. Therefore, a question-and/or risk-based approach based upon the mechanism of action and patient population was proposed as a reasonable TP-DI strategy. This field continues to evolve as companies generate additional preclinical and clinical data to improve their understanding of possible mechanisms for drug interactions. Regulatory agencies are in the process of updating their recommendations to sponsors regarding the conduct of in vitro and in vivo interaction studies for new drug applications (NDAs) and biologics license applications (BLAs).
\end{abstract}

KEY WORDS: drug interactions; question based; regulatory; risk based; therapeutic proteins.

\section{INTRODUCTION}

Therapeutic proteins (TPs) such as monoclonal antibodies (mAbs) are increasingly being combined with small molecule drugs (SMDs) and other TPs to treat various diseases including immune-mediated diseases and cancer. The Food and Drug Administration (FDA) recently high-

\footnotetext{
${ }^{1}$ Clinical Pharmacology, Department of Pharmacokinetics and Pharmacodynamics, gRED, Genentech, South San Francisco, California, USA.

${ }^{2}$ Pfizer Global Research and Development, New London, Connecticut, USA.

${ }^{3}$ Pfizer Global Research and Development, San Diego, California, USA.

${ }^{4}$ Office of Clinical Pharmacology, Office of Translational Sciences, Center for Drug Evaluation and Research, Food and Drug Administration, Silver Spring, Maryland, USA.

${ }^{5}$ The State University of New York at Buffalo, Buffalo, New York, USA.

${ }^{6}$ Merck \& Co., Inc., Whitehouse Station, New Jersey, USA.

${ }^{7}$ Centocor R\&D, Johnson \& Johnson, New Brunswick, New Jersey, USA.

${ }^{8}$ Amgen Inc., Thousand Oaks, California, USA.

${ }^{9}$ Biogen Idec, Weston, Massachussets, USA.

${ }^{10}$ Medical Products Agency, Uppsala, Sweden.

${ }^{11}$ To whom correspondence should be addressed. (e-mail: sandhyag@ gene.com)
}

lighted the potential and importance of therapeutic proteindrug interactions (TP-DI; 1,2). Other review papers have also recently been published on TP-DI (3-5). As TPs are not metabolized by cytochrome P450 (CYP) enzymes or transported by transporters and biotransformation studies have not been routinely conducted, it was assumed that TPs do not cause metabolism-based drug interactions (DI). However, recent reports suggest that the use of cytokines, cytokine modulators such as anti-cytokine mAb or immunosuppressant containing drug combinations, in inflammatory indications, can lead to CYP-mediated DI, with the SMD as the victim $(5,6)$. Many TPs, cytokines, or cytokine modulators (antagonists) have been shown to alter CYP or transporter activities through direct or indirect effects.

In vitro strategies for assessing TP-DI during drug development are limited. Because of inherent differences in metabolic pathways between TPs and SMDs, few preclinical or in vitro tools commonly used for DI assessment for SMDs can be readily adopted to predict DI for TPs. There are also constraints in designing appropriate clinical DI studies due to pharmacokinetic (PK) properties of TPs.

The FDA's Draft Drug Interaction Guidance published in 2006, titled "Drug Interaction Studies-Study Design, Data Analysis and Implications for Dosing and Labeling" states 
that classical biotransformation studies are not generally required for biologics because they are not metabolized by metabolizing enzymes (7). The guidance however raises concerns regarding potential interactions between TPs and SMDs such as interferons and SMDs or between two different TPs. The guidance also states that in vitro methods may not be suitable. Two recent publications from the FDA highlight the current perspectives on TP-DI, particularly those involving effect of cytokine modulators on CYPs $(1,2)$. The European Medicines Agency guidance published in July 2007 titled "Guideline on the Clinical Investigation of the Pharmacokinetics of Therapeutic Proteins" supports concerns about immunomodulators such as cytokines that have shown a potential for the inhibition or induction of CYP enzymes thereby altering the metabolism of SMDs metabolized by these enzymes (8).

It is critical to understand the possible DI mechanisms for TPs and build a strategy during drug development to ensure safe and effective use of therapeutics. An American Association of Pharmaceutical Scientists-sponsored workshop was organized $^{1,2}$ to address limitations and knowledge gaps in assessing the potential for TP-DI, to share drug development, research and regulatory experience in TP-DI assessment, and to develop strategies for assessing TP-DI during drug development. Participants included industry, academic, and regulatory representatives.

\section{GOALS AND OBJECTIVES}

This workshop aimed to provide participants with a clear understanding on how to develop strategies for assessing TPDI during drug development by:

- reviewing preclinical tools and in vitro test systems for assessing the DI potential of TPs such as cytokines and cytokine modulators,

- reviewing literature on clinically relevant TP-DI,

- discussing study designs and acceptance criteria for assessing PK- and pharmacodynamic (PD)-based TPDI in clinical studies, and

- providing participants with the knowledge and skills to develop a science driven approach for assessing the risk and potential of TP-DI.

This paper condenses the salient points, considerations, and positions presented and discussed during the workshop providing a sense of the state-of-the-art with respect to TP-DI exploration.

\footnotetext{
${ }^{1}$ Programming Committee: Sandhya Girish, Ph.D., Genentech, Inc., Co-Chair; Amita Joshi, Ph.D., Genentech, Inc., Co-Chair; Steven W Martin, Ph.D., Pfizer Inc.; Mark C Peterson, Ph.D., Biogen Idec; Lei Zhang, Ph.D., US Food and Drug Administration; Hong Zhao, Ph. D., US Food and Drug Administration

${ }^{2}$ Moderators and Speakers: Sandhya Girish, Genentech, Inc.; Steven W. Martin, Ph.D., Pfizer Inc.; Joseph Balthazar, Ph.D., University at Buffalo, The State University of New York; Raymond Evers, Ph.D., Merck \& Co., Inc.; Honghui Zhou, Ph.D., FCP, Centocor R\&D, Johnson \& Johnson; Lei Zhang, Ph.D., US Food and Drug Administration; Hong Zhao, Ph.D., US Food and Drug Administration; Min Zhu, Ph.D., Amgen Inc.; Amita Joshi, Ph.D., Genentech, Inc.; Lewis Klunk, Ph.D., Biogen Idec; Chao Han, Ph. D., Centocor R\&D, Johnson \& Johnson; Eva Gil Berglund, Ph.D., Medical Products Agency, Uppsala, Sweden; Shiew-Mei Huang, Ph. D., US Food and Drug Administration
}

\section{SESSION I: PROLOG-IN VITRO AND PRECLINICAL MODELS AND CURRENT STATUS}

\section{Preclinical Tools and In Vitro Test Systems to Assess TP-DI Potential during Drug Development}

In vitro studies with isolated human hepatocytes or liver microsomes generally provide insight into the PK DI potential for co-administered SMDs. In contrast, it is currently not feasible to predict the propensity for DI between TPs and SMDs. Although the effects in general have been weak to moderate, examples of DI between TPs and SMDs have been observed, particularly for cytokines. Based on clinical data with interferons and interleukins (913), two important conclusions can be drawn: (1) cytokines can cause the downregulation of a wide range of CYP or isoform specific CYP enzymes, (2) a high inter-individual variability in effects on CYP levels is observed. Complicating factors in interpreting clinical DI data with cytokines include: (1) variability in the dose and duration of treatment, (2) whether the study was conducted in healthy volunteers or in patients, and (3) use of non-standardized probe substrates to monitor CYP activities.

In contrast to the straightforward evaluation of in vitro hepatocyte data for SMD DI, in vitro TP DI data have proven to be more difficult to interpret (2). For example, although a high dose of interleukin (IL)-2 has shown decreased CYP3A4 and other CYP activities in human liver (12), this finding could not be reproduced in vitro using hepatocytes. However, a sustained downregulation was observed in hepatocytes cocultured with Kupffer cells (14), suggesting that IL-2 causes Kupffer cells to secrete a cytokine, which indirectly downregulates CYP3A4 in hepatocytes. Complex signaling may be often involved in TP-DI and it is not currently possible to assure that cell culture systems provide the appropriate mix of cell types required for meaningful evaluation in vitro. As such, relatively little is known about the effects of TPs on CYPs, and little is known about the effects of TPs on other drug metabolizing enzymes or drug transporters. Recent work has demonstrated that TNF- $\alpha$ and IL- 6 reduce the protein levels and activity of several drug transporters, supporting the contention that there is a clear need for additional investigation (15).

The observation that different cytokines can have differential effects on the various CYP enzymes suggests that there are multiple mechanisms which could result in reducing enzyme activities (16).

With current knowledge and technology, it is not feasible to make prospective predictions based on in vitro hepatocyte data whether cytokines have the propensity to cause DI with SMDs. An important step towards establishing whether this might be possible in the future would require systematic retrospective analysis using standardized assay conditions, and the identification of suitable positive controls to monitor assay performance.

\section{A Recent Biotechnology Industry Organization (BIO) Survey on TP-DI Evaluation during Development of TPs}

Because of emerging science and lack of clear regulatory guidance, there is a perception that substantial inconsistencies 
exist in DI assessment strategies for TPs across pharmaceutical/biotechnology companies. Recently, the BioSafe Pharmacokinetics and Disposition Expert Working Group completed a survey to better understand "current practices" as to when a DI assessment strategy should be incorporated into TP drug development programs. Twenty-one companies representing a comprehensive cross-section of the BIO organization participated in the survey. Unlike SMD-DI studies, in vitro and preclinical methods are not widely used for DI assessments of TPs. As a result, clinical methods are still primarily used for these assessments. Three methods are commonly employed: (1) dedicated DI studies, (2) population pharmacokinetics-based assessments, and (3) clinical cocktail studies. Survey results indicated that most of the observed DI involving TPs was not clinically relevant, i.e., did not result in dose adjustment. In several companies, science-driven, risk-based strategies are being applied to incorporate DI assessments for TP drug development. However, it is unclear from the survey whether other companies are currently developing similar strategies or intend to do so in the future. A future follow-up survey will attempt to address these topics, among others.

\section{SESSION II: INDUSTRY PERSPECTIVES ON TP-DI EVALUATION: CASE STUDIES AND STRATEGIES}

To evaluate current practices in industry, speakers from four companies with diverse experience in developing TPs presented challenges and strategies in assessing TP-DI with case examples and insights on how approaches were developed to address risk-based assessment of TP-DI. Examples were largely restricted to $\mathrm{mAbs}$ due to limited information available on other classes of TPs.

\section{Amgen's Integrated Approach to Assess TP-DI in Cancer Treatment}

The safety profile of drug combinations is critical for balancing treatment benefit and risk in cancer patients. Four selected TPs approved for cancer treatment, namely bevacizumab, trastuzumab, panitumumab, and cetuximab were used as examples. A literature review of 80 journal articles and conference abstracts revealed that TP-DI potential was evaluated in only one third of the clinical trials. Most results were either negative for DI or showed less than $30 \%$ change in mean drug exposure with large intersubject variability. In general, TP exposure was not affected in the presence of SMDs. Based on the literature data on these four cancer TPs, no interaction has been reported between TPs and no TP dose adjustments have been warranted.

TP-DI evaluation is usually not a stand-alone study but rather a substudy evaluation conducted as part of ongoing phase $2 / 3$ clinical trials. As a result, conventional "wellcontrolled" DI study conditions (e.g., control diet, comedications, disease status, treatment options, dosing time, and schedule) are hardly preserved. TP PK data is usually less intensive than those with traditional study designs for SMDDI studies. The number of subjects may not be well balanced across treatment cycles or arms, and the number of evaluable subjects is also affected by unscheduled dose and sampling time due to dose interruption/reduction or patient discontin- uation. Challenges also exist in non-compartmental PK analysis as there are often a significant number of data exclusions. PK assessments are further complicated as many TPs in oncology have non-linear pharmacokinetics. Moreover, since a TP-alone arm is uncommon in an oncology setting, historical data are usually referenced in the TP-DI evaluation. Therefore, a scientifically sound and technically feasible integrated TP-DI evaluation strategy needs to be developed to address these challenges.

It is preferable to include TP-DI assessment in early phase $1 b / 2$ trials because they are relatively small in size and operational issues are more easily addressed. Importantly, the early TP-DI data can help establish development strategies and also guide later phase study designs. If any TP-DI signals are identified in small early trials, expanded assessment can be considered. One limitation of early assessment is that the optimal drug combination is often modified in later stages of development. Although testing TP-DI in late phase for product registration is possible, it increases the complexity of pivotal phase 3 trials and is thus likely to have more confounding factors that can contribute to difficulty in data interpretation.

Selecting interacting drugs for TP-DI investigation is mainly based on potential concomitant usage, PK and PD properties and the therapeutic window of selected drugs. For evaluating PK interaction, commonly used study designs include:

1. one-sequence crossover design (e.g., chemotherapy followed by chemotherapy+TP to test TP effect on chemotherapy; testing effect of chemotherapy on TP may need cross-study comparison);

2. parallel design (e.g., TP+chemotherapy vs. chemotherapy alone to test the effect of TP on chemotherapy; testing effect of chemotherapy on TP may need cross-study comparison) and

3. a one-way sequential phase 1b study (open label) staggered with a phase 2 (double blind) study.

Design selection should be based on the PK properties of the interacting drugs as well as the types of interaction.

TP-DI evaluation is usually conducted as an estimation study with 10-20 patients per arm in either a PK subgroup study of phase $2 / 3$ trial or in a phase $1 b$ study. Due to large inter-subject variability in exposure for TP in oncology, fully powered TP-DI studies for hypothesis test are uncommon. Depending on the pharmacokinetics, intensive PK sampling has been collected for the chemo-agents and relative sparse PK sampling for TPs due to their much longer half-lives. Combined intensive and sparse sampling schemes may be collected in cycle 1 and at steady state. To evaluate the clinical relevance of any change in exposure (e.g., AUC, $\mathrm{C}_{\max }$, or $\mathrm{C}_{\min }$ ), safety, $\mathrm{PD}$, and efficacy measures may be collected along with the PK samples. If an interaction is observed, clinical relevance should be evaluated in conjunction with safety and efficacy data and should be the primary consideration in data interpretation.

Non-compartment analysis and/or population modeling approaches may be applicable to TP-DI evaluation. Covariate analysis may be used to identify potential contributing factors to drug exposure, as well as testing for study effects in crossstudy comparisons. The importance of the DI will be determined by the therapeutic index of the drug combination. 
Amgen found that developing an internal TP-DI database is extremely useful to aid systematic planning in design of TPDI studies across programs. For example, using a standardized approach to collect PK data for a specific chemotherapy regimen (e.g., paclitaxel) from control arms in different studies was extremely useful in the interpretation of results in new TP-drug combination studies.

Amgen thus presented an integrated approach as used in an oncology setting with specific details around timing of such a study, type of DI studies based on stage of drug development and DI signal in early studies. Modeling- and simulation-based TP-DI evaluation was used to address specific challenges.

\section{Genentech's Question- and Risk-Based Approach to Assess TP-DI}

The Genentech presentation provided an overview of potential mechanisms for TP-related DI, both PK and PD examples focused on mAb therapeutics, one of many TP classes. This approach may also be applied to other TPs. The proposed question- and risk-based approach assesses both effect of other therapeutics on TPs and effect of TPs on other drugs.

A systematic approach to answering the question of "Can a therapeutic $\mathrm{mAb}$ be either a victim or a perpetrator of a TP-DI?" assesses the clearance mechanisms of a particular $\mathrm{mAb}$ and asks whether extrinsic factors such as co-administered TPs or SMDs can affect these clearance processes. These clearance mechanisms can be classified under the following categories:

Specific mechanisms governed by target biology-these are typically high affinity, low capacity saturation processes like binding of mAbs to its target and subsequent targetmediated clearance. The biology of the target, including information on the site of expression, relative abundance of expression, and the pharmacology of the target are all important in assessing whether a TP or SMD in combination with a mAb can alter $\mathrm{mAb}$ pharmacokinetics. Specific examples of such PD interactions where target biology is altered are efalizumab in combination with triple immunosuppressant therapy (17) and anakinra in combination with etanercept (18). Both combinations resulted in PD-based interactions. In contrast, no alteration in target biology was anticipated and no interaction was noted for bevacizumab in combination with erlotinib (19).

Non-specific mechanisms that are typically governed by immunoglobulin structure are high-capacity, largely nonsaturable processes like neonatal $\mathrm{Fc}$ receptor (FcRn) recycling, non-specific uptake by tissues, binding to Fc $\gamma$ receptors, and proteolytic degradation. There is a high expression of FcRn and Fc $\gamma$ receptors, and theoretically very high concentrations of $\mathrm{IgG}$ can saturate the FcRn receptors. However, the concentration required is far higher than circulating $\mathrm{IgG}$ levels and therapeutic levels of mAbs (for example, endogenous $\mathrm{IgG}$ levels in normal individuals are about $10 \mathrm{mg} / \mathrm{mL}$ while therapeutic levels of mAbs are in microgram per milligram range and at these concentrations FcRn is not saturable). To date, there appears to be limited evidence of a DI mediated by non-specific clearance mechanism for mAbs in combination. Specific examples of $\mathrm{mAb}$ combinations where no alteration in PK was observed are trastuzumab combined with bevacizumab (20) and rituximab combined with bevacizumab (21). However, it is worth noting that a downregulation of Fc $\gamma$ receptors by methotrexate (MTX) is observed in patients with rheumatoid arthritis (RA). It is plausible that the change in Fc $\gamma$ receptors in the presence of MTX may affect $m A b$ clearance during $m A b$ treatment in combination with MTX in RA patients $(22,23)$.

Immunogenicity to TP may enhance clearance from the body. Some evidence exists suggesting an impact of immunosuppressive agents on $\mathrm{mAb}$ pharmacokinetics, possibly due to alteration in target (expression or cell number) and/or reduced immunogenicity. An apparent DI (i.e., decrease in mAb clearance) was noted when a combination with MTX was noted for infliximab (23) and adalimumab (24). This was attributed to MTX's immunosuppressive effect, decreasing immune response to the mAb. Additionally, the immunogenicity of anti-TNF- $\alpha$ agents in autoimmune diseases has been recently reviewed (25).

Tertiary carbohydrate structure can theoretically mediate clearance through carbohydrate-specific clearance mechanisms. However, no specific example of TP-DI has been cited for this possible mechanism.

TPs such as cytokines and cytokine modulators are likely to affect the pharmacokinetics of a SMD. Therefore, special attention should be given to narrow therapeutic range (NTR) drugs in combination with cytokine/cytokine modulators. Two additional categories of DI following combination of cytokines and cytokine modulators with SMDs are further discussed below:

1. TP is a cytokine or a cytokine modulator and therefore affects SMD metabolism (1). When muromonab and Cyclosporin A (CsA) (26) were given in combination there was an increase in CsA trough levels. The suggested mechanism for this is T-cell activation and subsequent release of multiple cytokines such as IL-2, IL-3, TNF $\alpha$, and TNF $\gamma$. Similarly, an increase in trough level of tacrolimus was observed in combination with basiliximab (27) and an increase in CsA levels when combined with basiliximab (28). For both of these interactions, an indirect mechanism is suggested. Binding of the mAb to IL-2R on activated Tcells may alter circulating IL-2Rs on hepatic and intestinal cells and could possibly result in downregulation of CYP3A4 enzyme activity. Potential DI mediated by such indirect mechanisms is a concern and currently, precautionary language exists in United States Prescribing Information for cytokine modulators, rilonacept, canakinumab, golimumab, and ustekinumab (29-32).

2. Drug-disease interaction is a mechanism whereby treatment with a cytokine or cytokine modulator could normalize previously downregulated CYP enzymes. This is illustrated conceptually by Morgan (6) and has been demonstrated in an in vivo DI study for tocilizumab + omeprazole and tocilizumab + simvastatin drug combinations but not for tocilizumab+ dextromethorphan (33). A decrease in omeprazole $(28 \%)$ and simvastatin $(57 \%)$ exposure was observed when combined with tocilizumab.

As also highlighted by the Amgen speaker that there are considerable logistical constraints and operational 
challenges when assessing TP-DI in the clinical setting especially in oncology. Therefore, dedicated DI studies in patients are not always possible. A dedicated TP-DI study was conducted to study irinotecan+bevacizumab when a DI alert was generated in a phase 3 study with this combination. The dedicated DI study (34) however showed that there was no effect of bevacizumab on irinotecan or SN-38 levels. In other clinical studies as well, to date, no PK interactions have been observed between bevacizumab and any of the following agents: capecitabine, cisplatin, 5fluorouracil, irinotecan, oxaliplatin, paclitaxel, interferon alfa-2a, or trastuzumab.

On the practical level, when applying this historical knowledge to evolve a TP-DI strategy for a mAb in development, it is clear that an individualized and iterative approach is warranted. Genentech's question-based risk assessment DI strategy as applied to therapeutic mAbs in development is summarized in Fig. 1. This process is incorporated into the clinical pharmacology plan in the early stages of development and utilizes a scientific approach to assessing potential mechanisms for TP-DI risk in the context of type of molecule, indication, pharmacokinetics, pharmacodynamics, study design considerations, immunogenicity, drug combinations, therapeutic index of drug, etc. A thorough knowledge of all of the aforementioned components will help determine the need for DI assessment and for data collection on a case-by-case basis when a $\mathrm{mAb}$ is combined with a SMD and/or with another $\mathrm{mAb}$. If DI assessment is scientifically reasonable, the risk assessment strategy is expected to guide the design and conduct of the appropriate study.

As illustrated in Fig. 1, Genentech may conduct DI analyses in phase $1 \mathrm{~b}$ or 2 or phase 3 clinical studies for select new molecular entities if the results of a DI risk assessment leads Genentech to believe that a safety or efficacy risk exists for a treatment combination. For molecules exhibiting a clinically relevant DI signal, addi- tional confirmatory analyses may be conducted in phase 3 studies.

\section{Risk-Based Approach to Assess Potential for TP-DI in Biogen Idec}

When considering DI, maintaining patient safety and drug efficacy is paramount. In addition to ethical concerns, clinical DI studies can add to the cost and timeline of drug development. Therefore, these studies should only be performed when there is a clear scientific rationale to do so. Figure 2 provides a flow chart for Biogen's risk-based approach to determine the need for interaction studies where the TP is the perpetrator and the SMD is the victim. If the $\mathrm{TP}$ is a cytokine or modulates a cytokine which can effect regulation of various CYPs, and these CYPs provide the primary clearance pathway for the coadministered SMD, then a clinical DI study to examine potential interaction should be considered. Consideration should also be given to whether the SMD has a NTR or required therapeutic drug monitoring. If these conditions are not met, there is no clear scientific rationale for performing a DI study. Likewise, for other TPs such as mAbs, there must be a clear mechanistic rationale for a potential interaction with an SMD; otherwise, no interaction study should be needed. An adequately powered population PK approach should also be considered (this may be more applicable to the mAb stream in the decision tree).

This "risk-based" approach is science driven, eliminates the need for unnecessary or low value clinical studies, reduces cost and time of drug development, and is expected to satisfy DI requirements. For TP-SMD combinations where a DI study is indicated, bioanalytical resources can be optimized by storing samples for analysis until a proof of concept is established. Biogen's "collect and hold" strategy for assessing DI is somewhat unique.

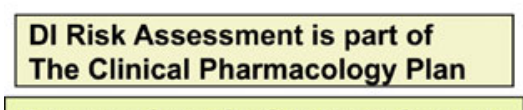

Program Specific Strategic Context
Can a TP affect SMD clearance?

- Can the TP affect CYP450 enzymes?

If yes, is it given with NTR drugs?

What can affect $\mathrm{mAb}$ clearance?

- Can external factors change Target Biology?

- Can immune reaction to foreign protein affect the $\mathrm{mAb} C L$ ?

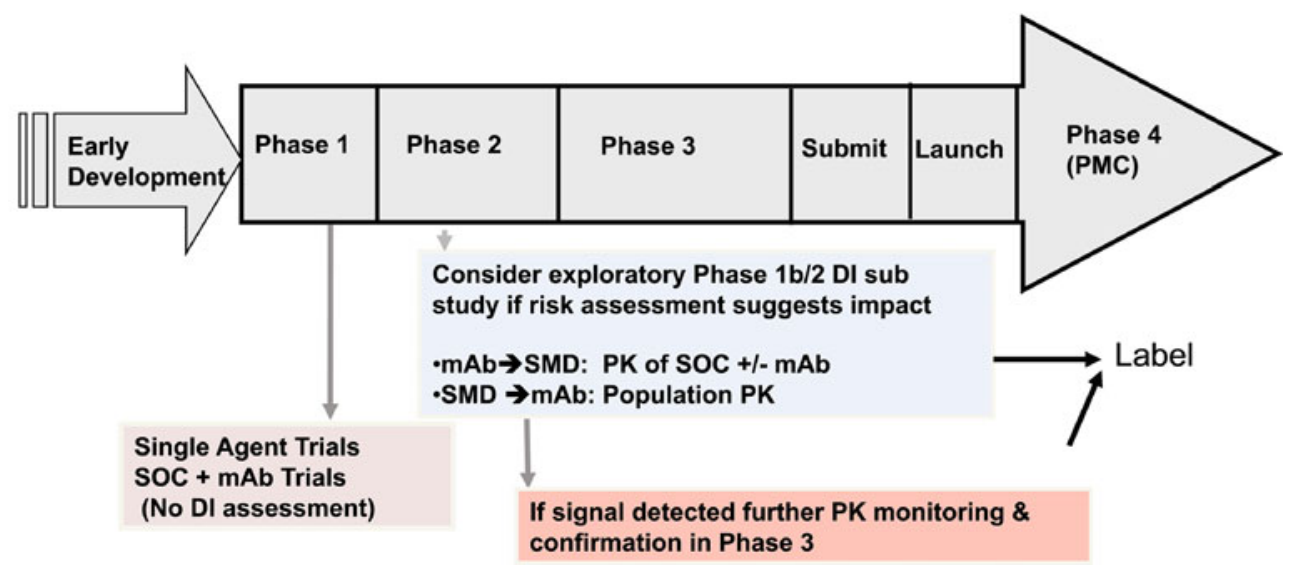

Fig. 1. Genentech's TP-DI strategy as applied to a mAb during drug development ( $D I$ drug interactions, $T P$ therapeutic protein, SMD small molecule drug, CYP450 cytochrome P450, CL clearance, NTR narrow therapeutic range, $m A b$ monoclonal antibody, $P M C$ post-marketing commitment, $S O C$ standard of care) 


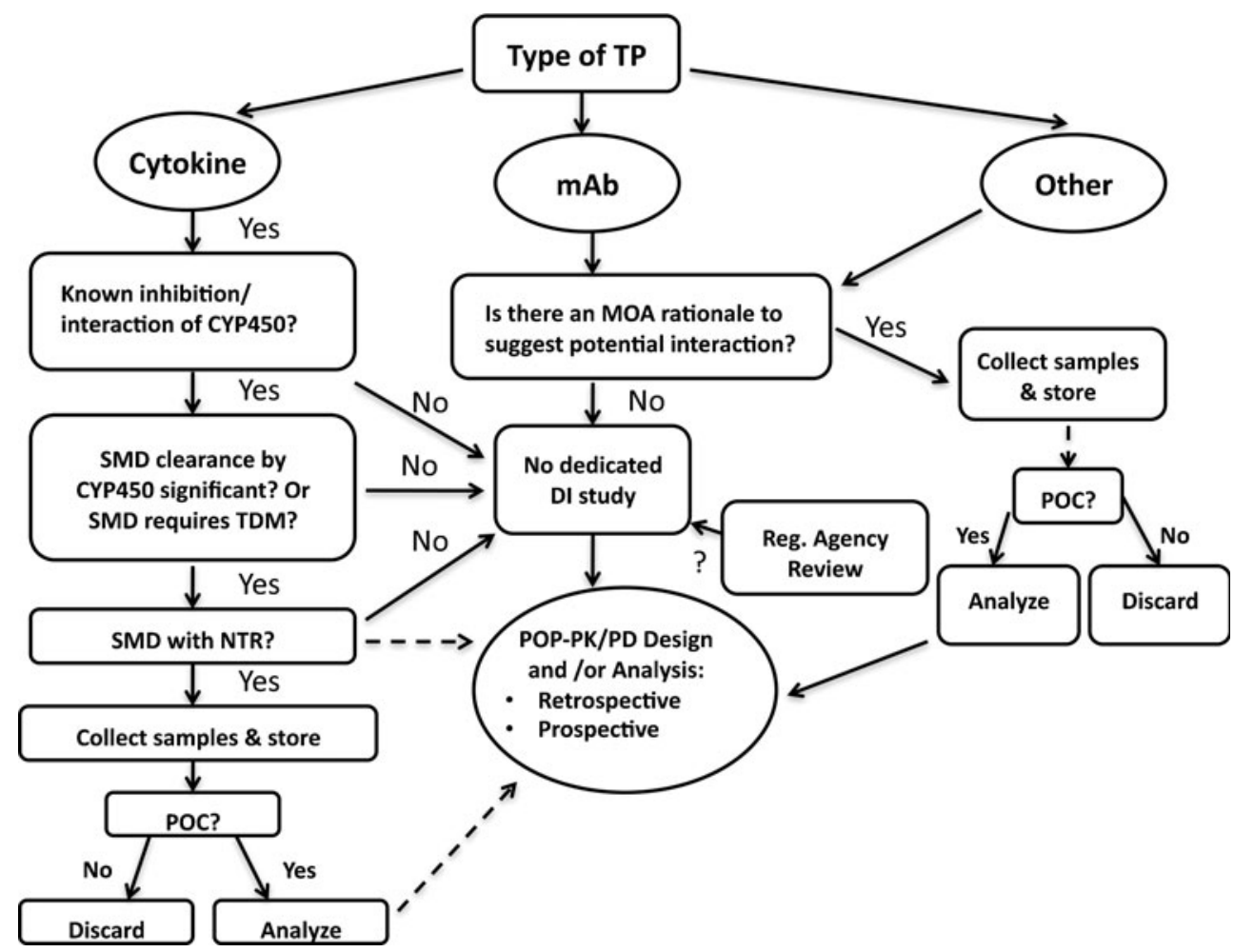

Fig. 2. Biogen Idec's risk-based approach to assess potential for TP-DI. (TP therapeutic protein, $m A b$ monoclonal antibody, MOA mechanism of action, SMD small molecule drug, CYP450 cytochrome P450, $T D M$ therapeutic drug monitoring, $D I$ drug interactions, $P O C$ proof of concept, $N T R$ narrow therapeutic range, $P O P-P K / P D$ population-PK/PD)

\section{Centocor's Strategy to Address TP-DI}

The presentation briefly reviewed the pathways for disposition of SMDs and mAbs to explore plausible mechanisms of interaction and discussed technical considerations and strategic approaches taken at Centocor. As there is no global strategy available to address potential TP-DI and no regulatory guidance for preclinical and clinical assessment, Centocor has adopted a risk-based strategy for the assessment of PK-based mAb-DI (Fig. 3). The integrated risk assessment is designed to utilize existing data and knowledge, maintain clinical relevance, and be alert and open to new information. A framework is built based on several key factors such as the therapeutic target, therapeutic window of the agents, and plausible mechanisms. Preclinical and

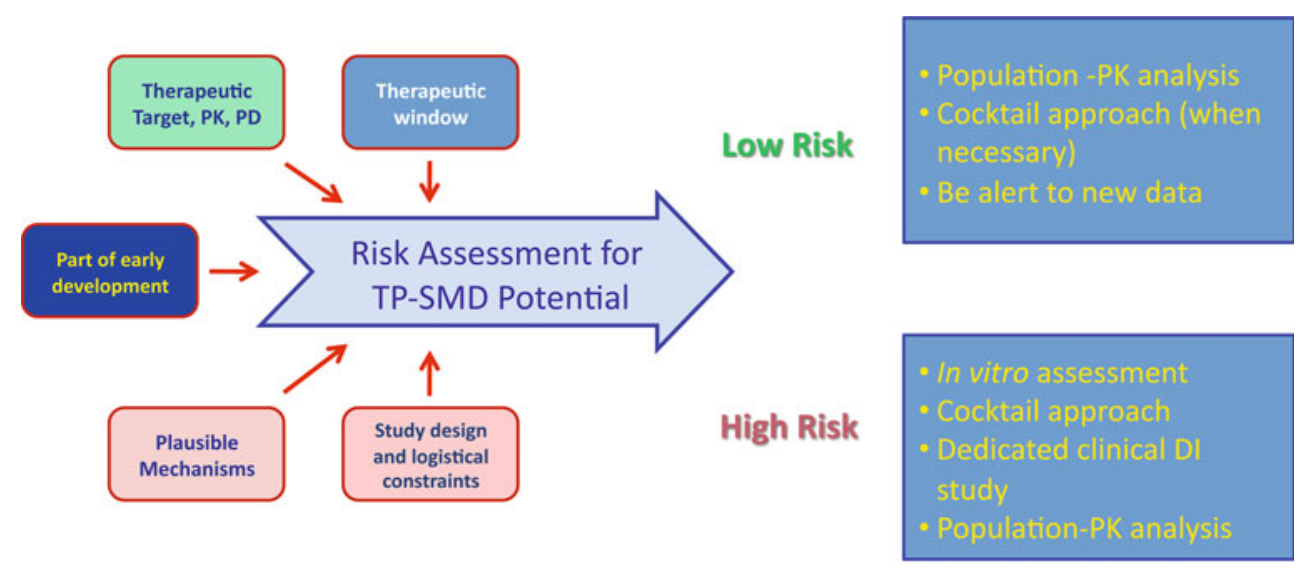

\section{Development of science and technology in the area}

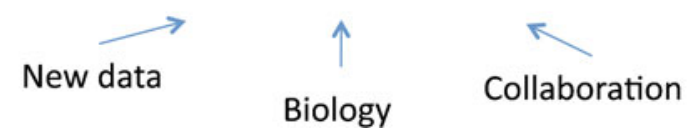

Fig. 3. Centocor's strategy to address TP-DI (TP-SMD therapeutic protein-small molecule drug interaction, $P K$ pharmacokinetic, $P D$ pharmacodynamic, $D I$ drug interactions) 
clinical studies for the assessment of potential mAb-DI are conducted on a case-by-case basis. Potential applicable approaches encompass population PK modeling based assessment, dedicated or "cocktail" clinical studies, and mechanismbased in vitro evaluation.

\section{SESSION III: REGULATORY PERSPECTIVES}

\section{European Regulatory Perspective on TP-DI Strategy}

The current European Guideline on the investigation of DI was adopted a decade ago. In April 2010, a draftrevised guideline was adopted by the Committee for Medicinal Products for Human Use (CHMP) for release on a 6-month public comment period (35). The draft guideline does not contain advice related to TPs. This was intentional as it was considered difficult to give detailed advice based on the available knowledge. However, some advice is given in another specific guideline "The CHMP guideline on the clinical investigation of the pharmacokinetics of therapeutic proteins" published in 2007 (8). This guideline states that some TPs have been found to cause DI and that the need for DI studies should be considered on a case-by-case basis. Interaction studies should be considered when the protein induces changes to elimination pathways (receptors) also involved in the elimination of other drugs or when suppression of the immunological system is likely, as also when there are possible interactions with endogenous proteins as well as interactions due to effect on target (e.g., receptor) expres- sion. It is pointed out that the interaction studies generally need to be of multiple-dose design.

The DI at an enzyme level observed so far has generally been of a moderate magnitude. The effects seem mainly relevant for NTR drugs but some increases in drug clearance found could also be of clinical relevance for other medicinal products. In some cases, the effect observed in patients is actually a normalization of enzyme expression. This makes translating the effects into practically applicable labeling advice difficult unless the affected drug is individually titrated. The recommendation may need to be different depending on whether the affected drug was initiated before or after the manifest of the disease.

The optimal study design of a TP-DI study is likely to be a long duration in vivo investigation, possibly with repeated DI measurements in the target patient population. Due to the challenges of performing such a study, in vitro methods which may detect the interactions are warranted as general screening tools. Possibly, cultured hepatocytes with verified presence of enzyme regulatory systems via PXR, CAR, and NF-k $\beta$ could be used for effects via these pathways. However, other interaction mechanisms may exist, which then also needs verification during the culturing. Other in vitro methods to investigate the effect on interleukins may be a way forward to screen for interaction potential of this specific mechanism. However, due to the lack of experience with these methods, it is possible that the results may only be used qualitatively.

According to the labeling of the TPs registered in the last 5 years on the EU market, PK interaction studies were not performed with majority of the drugs. Slightly more

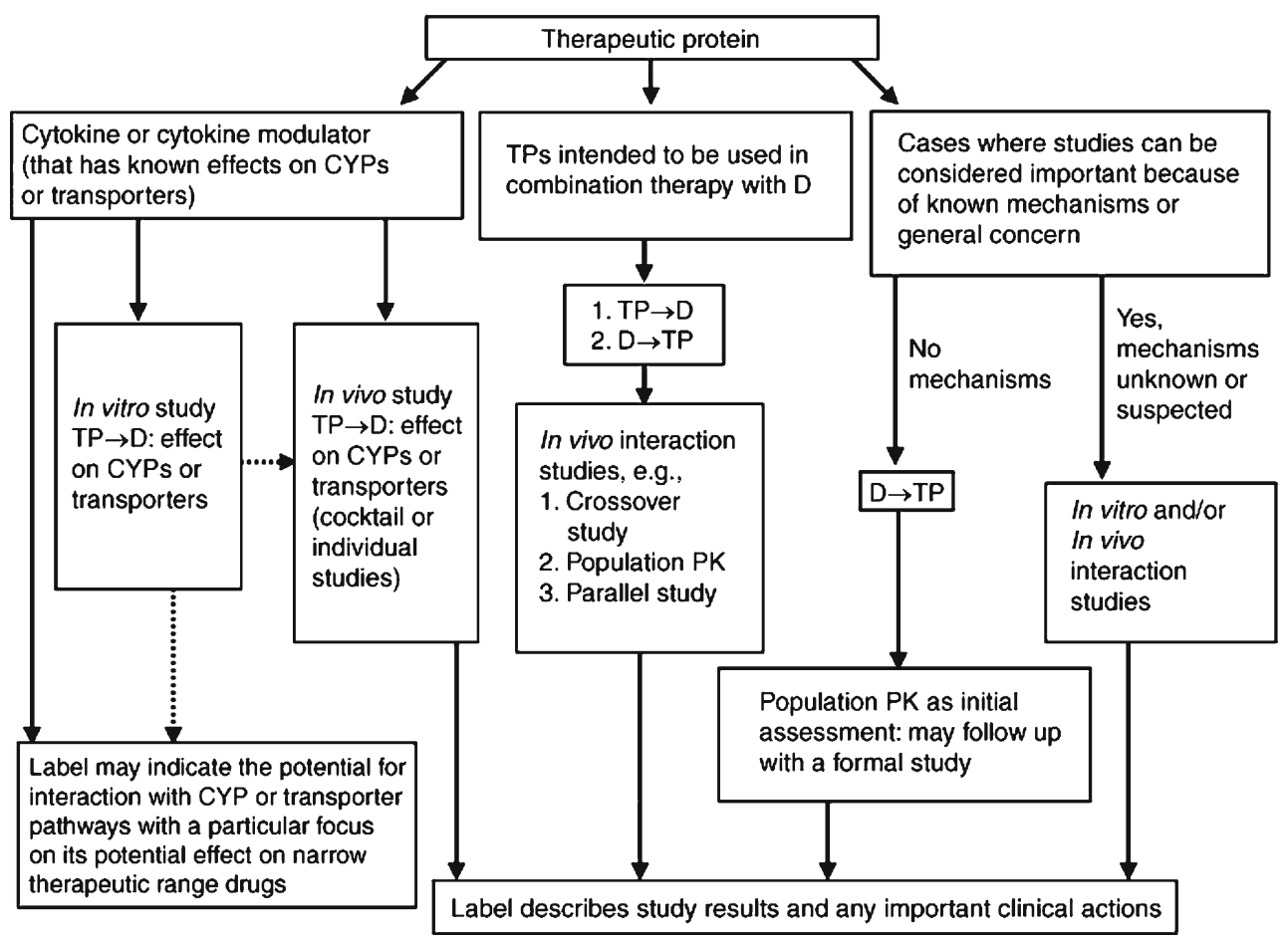

Fig. 4. TP-DI and implications for drug development (2). Summary of the types of studies that have been used during drug development to evaluate therapeutic protein (TP)-small molecule drug (D) interactions. This includes an evaluation of the effect of TP on $\mathrm{D}(\mathrm{TP} \rightarrow \mathrm{D})$ and the effect of $\mathrm{D}$ on TP $(\mathrm{D} \rightarrow \mathrm{TP})$. The broken lines suggest the limited use of in vitro studies for informing in vivo study design or labeling (CYP cytochrome $\mathrm{P} 450$, population $P K$ population pharmacokinetics) 
Table I. Summary of Drug Interaction Studies in the US FDA Approved Package Inserts for New Molecular Entity TPs Approved by the End of February 2010 (Adapted from Reference 1)

\begin{tabular}{lllll}
\hline Category & Dedicated studies & Some information & No information & Total (\%) \\
\hline Cytokines & 2 & 7 & 2 & $11(14)$ \\
Growth factors & 0 & 2 & 8 & $10(13)$ \\
Enzymes & 1 & 7 & 9 & $17(22)$ \\
Monoclonal antibodies & 6 & 13 & 10 & $29(38)$ \\
Miscellaneous & 0 & 6 & 3 & $9(12)$ \\
Total & $9(12 \%)$ & $35(46 \%)$ & $32(42 \%)$ & $76(100)$ \\
\hline
\end{tabular}

Reproduced from (1) with permission from Adis, a Wolters Kluwer business (๔ Adis Data Information BV 2010. All rights reserved)

than one fifth of the TPs had a statement in the labeling that "Drug interactions were not expected". A small percentage of the approved drug applications contained results from conventional DI studies. However, in the labeling of almost one tenth of the products, it was stated that effects on CYP enzymes were expected although not studied. Another tenth included information from population PK analyses.

\section{Evolution of Regulatory Guidance Development by the US Food and Drug Administration for Small Molecules and Application to Large Molecule DI}

Various intrinsic and extrinsic factors can affect an individual patient's drug exposure and response (36). The US FDA has published several guidance documents recommending how and when to evaluate these factors, including DI, during drug development (37). The need to evaluate DI during drug development cannot be over- emphasized. Metabolism- and transporter-based DI have contributed to adverse events with some drugs that were withdrawn from the US market. Drug labels need to and have been updated to include information related to DI. Recent examples include clopidogrel: its interaction with CYP2C19 inhibitors and the use in patients with deficient CYP2C19 enzyme activities have been recently added to its labeling. In addition, developing tools (in vitro methodologies and pharmacogenetics evaluation) to evaluate the role of various ATP-binding cassette and solute carrier transporters in drug disposition (38) and thereby safety and efficacy have increased understanding of DI involving statin drugs [e.g., rosuvastatin and organic anion transporting polypeptide 1B1 (OATP1B1) and breast cancer resistance protein]. The US FDA has proposed various decision trees to help determine when to evaluate transporter-based DI during drug development (39). Further, TP-DI studies have been increasingly conducted (Fig. 4) and included in the labeling (2). Continued evaluation of their clinical significance will help the US

Table II. Examples of Labeling Dealing with TP-DI (that Result in Pharmacokinetic Changes)

Drug name
1. CYP enzyme modulation by cytokines
and cytokine modulators
Tocilizumab (Actemra)

2. Immunosuppressive effect Infliximab (Remicade)

3. Mechanisms to be elucidated
Palifermin (Kepivance)

Trastuzumab (Herceptin)

Labeling language

7 Drug interactions
7.2 Interactions with CYP450 substrates

The effect of tocilizumab on CYP enzymes may be clinically relevant for CYP450 substrates with narrow therapeutic index, where the dose is individually adjusted. Upon initiation or discontinuation of ACTEMRA, in patients being treated with these types of medicinal products, therapeutic monitoring of effect (e.g., warfarin) or drug concentration (e.g., cyclosporine or theophylline) should be performed and the individual dose of the medicinal product adjusted as needed. Prescribers should exercise caution when ACTEMRA is coadministered with CYP3A4 substrate drugs where decrease in effectiveness is undesirable, e.g., oral contraceptives, lovastatin, atorvastatin, etc. The effect of tocilizumab on CYP450 enzyme activity may persist for several weeks after stopping therapy [see Clinical Pharmacology (12.3)]

\footnotetext{
7 Drug interactions

7.3 Methotrexate (MTX) and other concomitant medications

Concomitant MTX use may decrease the incidence of anti-infliximab antibody production and increase infliximab concentrations

\section{Drug interactions}

Avoid co-administration of palifermin with heparin. If heparin is used to maintain an intravenous line, rinse the line with saline prior to and after Kepivance administration 7 Drug interactions

In clinical studies, administration of paclitaxel in combination with Herceptin resulted in a 1.5fold increase in Trastuzumab serum levels [see Clinical Pharmacology (12.3)]
} 
FDA in formulating its recommendations in this area. Recent advancements in the development of physiologically-based PK modeling for SMDs has been critical and has been used to help address questions including in vitro to in vivo extrapolations and simulations of the extent of DI under various dosing regimens of the interacting drugs, in patients with concomitant renal impairment, in patients with various genotypes of metabolizing enzymes, etc. (40). Successful collaboration between academia, industry, and government agencies in this field and in other areas, illustrated by the establishment of the International Transporter Consortium for developing a

Table III. Examples of Labeling Dealing with TP-DI (that Result in Pharmacodynamic Changes)

1. Growth factors (Palifermin and G-CSF) and chemotherapy

Palifermin (Kepivance)

Drug interactions

Do not administer Kepivance within $24 \mathrm{~h}$ before, during infusion of, or within $24 \mathrm{~h}$ after administration of myelotoxic chemotherapy [see Dosage and Administration (2.2) and Clinical Studies (14)]. In a clinical trial, administration of Kepivance within $24 \mathrm{~h}$ of chemotherapy resulted in increased severity and duration of oral mucositis

Filgrastim (Neupogen)

Precautions

Because of the potential sensitivity of rapidly dividing myeloid cells to cytotoxic chemotherapy, do not use NEUPOGEN® in the period $24 \mathrm{~h}$ before through $24 \mathrm{~h}$ after the administration of cytotoxic chemotherapy (see Dosage and Administration)

Pegfilgrastim (Neulasta)

NEULASTA ${ }^{\circledR}$ should not be administered in the period between 14 days before and $24 \mathrm{~h}$ after administration of cytotoxic chemotherapy (see Dosage and Administration) because of the potential for an increase in sensitivity of rapidly dividing myeloid cells to cytotoxic chemotherapy

2. Growth factors and myeloablative therapy Oprelvekin (Neumega)

Warnings (Neumega)

Increased toxicity following myeloablative therapy

Neumega is not indicated following myeloablative chemotherapy. In a randomized, placebo-controlled phase 2 study, the effectiveness of Neumega was not demonstrated (see Clinical Studies, Study in Patients Following Myeloablative Chemotherapy). In this study, a statistically significant increased incidence in edema, conjunctival bleeding, hypotension, and tachycardia was observed in patients receiving Neumega as compared to placebo.

The following severe or fatal adverse reactions have been reported in post-marketing use in patients who received Neumega following bone marrow transplantation: fluid retention or overload (e.g., facial edema, pulmonary edema), capillary leak syndrome, pleural and pericardial effusion, papilledema and renal failure

3. Increased toxicities with concurrent therapy (proleukin and psychotropic, nephrotoxic, myelotoxic, cardiotoxic or hepatotoxic drugs)

Aldesleukin (Proleukin)

Drug interactions

PROLEUKIN may affect central nervous function. Therefore, interactions could occur following concomitant administration of psychotropic drugs (e.g., narcotics, analgesics, antiemetics, sedatives, tranquilizers)

Concurrent administration of drugs possessing nephrotoxic (e.g., aminoglycosides, indomethacin), myelotoxic (e.g., cytotoxic chemotherapy), cardiotoxic (e.g., doxorubicin) or hepatotoxic (e.g., methotrexate, asparaginase) effects with PROLEUKIN may increase toxicity in these organ systems. The safety and efficacy of PROLEUKIN in combination with any antineoplastic agents have not been established

In addition, reduced kidney and liver function secondary to PROLEUKIN treatment may delay elimination of concomitant medications and increase the risk of adverse events from those drugs

4. Increased infections with concurrent therapy Etanercept (Enbrel)

Drug Interactions

In a study in which patients with active Rheumatoid Arthritis were treated for $\leq 24$ weeks with concurrent Enbrel and anakinra therapy, a $7 \%$ rate of serious infections was observed, which was higher than that observed with Enbrel alone $(0 \%)$. Two percent of patients treated concurrently with Enbrel and anakinra developed neutropenia (ANC $<1 \times 109 / 1)$

5. Increased myelotoxicity (interferon alfa-2b and myelosuppressive agents)

Interferon alfa-2b (Intron A) Drug interactions

Caution should be exercised when administering INTRON A therapy in combination with other potentially myelosuppressive agents such as zidovudine 
white paper for transporters (38), are keys to success in developing useful information for the safe and effective use of SMDs (41). It is intended that similar efforts be applicable to TP-DI.

\section{Regulatory Perspectives on TP-DI Evaluation-Case Studies}

Table I summarizes DI studies included in the US FDAapproved package inserts for new molecular entity TPs approved by the end of February 2010 (1). Various possible mechanisms of PK TP-DI were presented with examples and labeling impact (Table II).

1. CYP enzyme modulation by cytokines and cytokine modulators

Tocilizumab was used as an example to illustrate the effect of cytokine modulators on CYP enzymes. CYP enzymes in the liver are downregulated by infection and inflammation stimuli including cytokines such as IL-6. Inhibition of IL-6 signaling in RA patients treated with tocilizumab may restore CYP activities to higher levels than those not treated with tocilizumab leading to increased metabolism of drugs that are CYP substrates. As stated earlier in this paper, in vivo studies showed that the exposure of omeprazole (a substrate of CYP2C19 and CYP3A4) and simvastatin (a substrate of CYP3A4 and transporter OATP1B1) decreased 1 week following a single dose of tocilizumab.

2. Immunosuppressive effect

MTX, an immunosuppressive agent, reduced the apparent clearance of infliximab likely due to the reduced incidence of anti-infliximab formation. At 8 weeks after a maintenance dose of $3-10 \mathrm{mg} / \mathrm{kg}$ of infliximab, median infliximab serum concentrations ranged from approximately $0.5-6 \mathrm{mcg} / \mathrm{mL}$; however, infliximab concentrations were not detectable $(<0.1 \mathrm{mcg} / \mathrm{mL})$ in patients who tested positive for antibodies to infliximab (42). The incidence of antiinfliximab antibody was $21 \%$ and $7 \%$, respectively in the absence and presence of MTX at 12 weeks after last infliximab infusion at a $3 \mathrm{mg} / \mathrm{kg}$ dose (23).

3. Mechanisms to be elucidated

The mechanism for the observed fivefold increase in the systemic exposure of palifermin, a recombinant human keratinocyte growth factor, when it was coadministered with heparin, has not been fully elucidated. Also the mechanism for paclitaxel increasing trastuzumab systemic exposure is not clear (Table II).

Possible mechanisms of PD interactions were also discussed with labelling impact (Table III).

\section{CONCLUSIONS}

DI studies are regularly conducted with conventional SMDs, but few DI studies have been performed with TPs. In this workshop, industry, academic, and regulatory speakers highlighted important emerging themes for consideration in DI assessment for TPs such as: molecule type, target, indication, and disease biology. Speakers also discussed recent reports regarding the regulation of CYP enzymes and transporters in inflammatory states. The use of cytokines, cytokine modulators, and immunosuppressant-containing combinations in inflammatory indications may have potential for DI, in particular when TPs are combined with NTR drugs. A question- and risk-based (the definition of risk will be individualized) integrated approach depending on the mechanism of action of TPs and patient population appears to have evolved and is used within companies like Genentech, Biogen Idec, Amgen and Centocor. Critical knowledge gaps and areas needing further research were identified (Table IV). The US FDA is updating its recommendations to sponsors of new drug applications and biologics license applications who are conducting in vitro and/or in vivo interaction studies with TPs (7). This guidance is expected to address some of the challenges unique to biologics.

Table IV. Summary of Knowledge Gaps and Areas Needing Further Research

Knowledge gaps

Several knowledge gaps were identified that require further cross collaboration between academia, industry and regulators. While there is extensive literature on the effects of cytokines and cytokine modulators in $\mathrm{CYP}^{\mathrm{a}}$ enzyme levels $(1,2,5,16)$ there is a knowledge gap in our understanding of how in vitro assessment of cytokines and cytokine modulators alter CYP enzyme levels/activity and how this can translate to meaningful in vivo changes in exposure

- Determine appropriateness of in vitro systems to study in vivo TP-DI ${ }^{\mathrm{b}}$

- TP-DI mechanisms proposed need to be validated with actual in vitro data wherever possible

- Technical optimization and validation of in vitro systems for studying TP-DI

O Determine what systems/cell types are appropriate for studying TP-DI

O Develop methodology that is robust, reproducible and relevant to in vivo data

O Develop a more mechanistic understanding of how $\mathrm{TPs}^{\mathrm{c}}$ alter CYP activity in vitro and in vivo

$\bigcirc$ Understand whether other drug metabolizing enzymes (besides CYP) and transporters are involved in TP-DI

- Inflammation and effect on CYP

$\bigcirc$ Better understand how constant and intermittent inflammation could cause a change in cytokines and thereby affect CYP

Understand whether and how this can be translated into understanding TP-DI in the clinical setting

- Give priority to generate in vitro data to grow our knowledge base $\bigcirc$ Priority to (1) cytokines (2) TPs that are cytokine modulators

$\bigcirc$ Priority for studying existing proposed mechanisms of TP-DI

$\bigcirc$ Priority to understand various mechanism via which TPs could cause TP-DI

- Develop exploratory multiphase/physiological/systems biology models to study cytokine mediated effect on CYP

- Data analyses gap-assess best practice for including concomitant medications in assessing TP-DI via POP-PK ${ }^{\mathrm{d}}$

${ }^{a}$ CYP cytochromes $\mathrm{P} 450$

${ }^{b} T P-D I$ therapeutic protein-drug interactions

${ }^{c} T P S$ Therapeutic proteins

${ }^{d} P O P-P K$ population pharmacokinetics 


\section{ACKNOWLEDGMENTS}

The authors thank the sponsor and co-sponsors for their support. This workshop was sponsored by American Association of Pharmaceutical Scientists and co-sponsored by ISSX (International Society for Study of Xenobiotics), Drug Information Association (DIA), and Biotechnology Industry Organization (BIO).

\section{REFERENCES}

1. Lee JI, Zhang L, Men AY, Kenna LA, Huang SM. CYPmedicated therapeutic protein-drug interactions clinical findings, proposed mechanisms and regulatory implications. Clin Pharmacokinet. 2010;49(5):295-310.

2. Huang S-M, Zhao H, Lee JI, Reynolds K, Zhang L, Temple R, et al. Therapeutic protein-drug interactions and implications for drug development. Clin Pharmacol Ther. 2010;87:497-503.

3. Seitz K, Zhou H. Pharmacokinetic drug-drug interaction potentials for therapeutic monoclonal antibodies: reality check. J Clin Pharmacol. 2007;47:1104-18.

4. Zhou H, Davis HM. Risk-based strategy for the assessment of pharmacokinetic drug-drug interactions for therapeutic monoclonal antibodies. Drug Discov Today. 2009;14(17-18):891-8.

5. Mahmood I, Green MD. Drug interaction studies of therapeutic proteins or monoclonal antibodies. J Clin Pharmacol. 2007:47:1540-54

6. Morgan ET. Impact of infectious and inflammatory disease on cytochrome P450-mediated drug metabolism and pharmacokinetics. Clin Pharmacol Ther. 2009;85(4):434-8.

7. US Food and Drug Administration. Draft Guidance for industry: drug interaction studies - study design, data analysis and implications for dosing and labelling. Rockville, MD. 2006. http://www.fda.gov/ downloads/Drugs/GuidanceComplianceRegulatoryInformation/ Guidances/ucm072101.pdf or FDA Drug Interaction and Drug Development website: http://www.fda.gov/Drugs/Development ApprovalProcess/DevelopmentResources/DrugInteractions Labeling/ucm080499.htm. Accessed 04 Jan 2010.

8. European Medicines Agency. Guideline on the clinical investigation of the pharmacokinetics of therapeutic proteins CHMP/EWP/ 89249/2004. London, UK. 2007. http://www.ema.europa.eu/ema/ index.jsp?curl=pages/regulation/general/general_content_000370. jsp\&murl=menus/regulations/regulations.jsp\&mid=WC0b01a c0580032ec5. Accessed 15 Jan 2011.

9. Pageaux GP, Le Bricquir Y, Berthou F, Bressot N, Picot M-C, Blanc $\mathrm{F}$, et al. Effects of interferon-alpha on cytochrome P-450 isoforms $1 \mathrm{~A} 2$ and $3 \mathrm{~A}$ activities in patients with chronic hepatitis C. Eur J Gastroenterol Hepatol. 1998;10:491-5.

10. Becquemont L, Chazouilleres O, Serfaty L, Poirier JM, Broly F, Jailon $\mathrm{P}$, et al. Effect of interferon $\alpha$-ribavirin bitherapy on cytochrome P450 1A2 and 2D6 and N-acetyltransferase-2 activities in patients with chronic hepatitis C. Clin Pharmacol Ther. 2002;71:488-95.

11. Elkahwaji J, Robin MA, Berson A, Tinel M, Letteron P, Labbe $\mathrm{G}$, et al. Decrease in hepatic cytochrome P450 after interleukine2 immunotherapy. Biochem Pharmacol. 1999;57:951-4.

12. Gorski JC, Hall SD, Becker P, Affrime MB, Cutler DL, Haehner-Daniels B. In vivo effects of interleukin-10 on human cytochrome $\mathrm{P} 450$ activity. Clin Pharmacol Ther. 2000;67:32-43.

13. Islam M, Frye RF, Richards TJ, Sbeitan I, Donelly SS, Glue P, et al. Differential effect of IFN $\alpha-2 b$ on the cytochrome P450 enzyme system: A potential basis of IFN toxicity and its modulation by other drugs. Clin Cancer Res. 2002;8:2480-7.

14. Sunman JA, Hawke RL, LeCluyse EL, Kashuba AD. Kupffer cell-mediated IL-2 suppression of CYP3A activity in human hepatocytes. Drug Metab Dispos. 2004:32:359-63.

15. Vee ML, Lecureur V, Stieger B, Fardel O. Regulation of drug transporter expression in human hepatocytes exposed to the proinflammatory cytokines tumor necrosis factor- $\alpha$ or interleukin-6. Drug Metab Dispos. 2008;37:685-93.
16. Aitken A, Richardson TA, Morgan ET. Regulation of drugmetabolizing enzymes and transporters in inflammation. Annu Rev Pharmacol Toxicol. 2006;46:123-49.

17. Vincenti F, Mendez R, Pescovitz M, Rajagopalan PR, Wilkinson $\mathrm{AH}$, Butt $\mathrm{K}$, et al. A phase I/II randomized open-label multicenter trial of efalizumab, a humanized anti-CD11a, anti-LFA-1 in renal transplantation. Am J Transplant. 2007;7:1770-7.

18. Genovese MC, Cohen S, Moreland L, Lium D, Robbins S, Newmark R, et al. Combination therapy with etanercept and anakinra in the treatment of patients with rheumatoid arthritis who have been treated unsuccessfully with methotrexate. Arthritis Rheum. 2004;50(5):1412-9.

19. Herbst RS, Johnson DH, Mininberg E, Carbone DP, Henderson T, Kim ES, et al. Phase I/II trial evaluating the anti-vascular endothelial growth factor monoclonal antibody bevacizumab in combination with the HER-1/epidermal growth factor receptor tyrosine kinase inhibitor erlotinib for patients with recurrent non-small-cell lung cancer. J Clin Oncol. 2005;23(11):2544-55.

20. Pegram MD, Chan D, Dickmann RA. Phase II combined biological therapy targeting the HER2 proto-oncogene and vascular endothelial growth factor using trastuzumab $(\mathrm{T})$ and bevacizumab (B) as first line therapy of HER2 amplified breast cancer [abstract 301]. Breast Cancer Res Treat. 2006;100 suppl 1: S28.

21. Ganjoo KN, An CS, Robertson MJ, Gordon LI, Sen JA, Weisenbach J, et al. Rituximab, bevacizumab and CHOP (RACHOP) in untreated diffuse large B-cell lymphoma: safety, biomarker and pharmacokinetic analysis. Leuk Lymphoma. 2006;47(6):998-1005.

22. Wijngaarden S, van Roon JA, van de Winkel JG, Bijlsma JW, Lafeber FP. Down-regulation of activating Fcgamma receptors on monocytes of patients with rheumatoid arthritis upon methotrexate treatment. Rheumatology (Oxford). 2005;44 (6):729-34.

23. Maini RN, Breedveld FC, Kalden JR, Smolen JS, Davis D, Macfarlane JD, et al. Therapeutic efficacy of multiple intravenous infusions of anti-tumor necrosis factor $\alpha$ monoclonal antibody combined with low-dose weekly methotrexate in rheumatoid arthritis. Arthritis Rheum. 1998;41(9):1552-63.

24. Humira ${ }^{\circledR}$ (Adalimumab) [packet insert]. Chicago, IL. Abbott Laboratories; 2010.

25. Emi Aikawa N, de Carvalho JF, Artur Almeida Silva C, Bonfá E. Immunogenicity of anti-TNF- $\alpha$ agents in autoimmune diseases. Clin Rev Allergy Immunol. 2010;38:82-9.

26. Vasquez EM, Pollak R. OKT3 therapy increases cyclosporine blood levels. Clin Transplant. 1997;11(1):38-41.

27. Sifontis NM, Benedetti E, Vasquez EM. Clinically significant drug interaction between basiliximab and tacrolimus in renal transplant recipients. Transplant Proc. 2002;34:1730-2.

28. Strehlau J, Pape L, Offner G, Nashan B, Ehrich JH. Interleukin2 receptor antibody-induced alterations of ciclosporin dose requirements in paediatric transplant recipients. Lancet. 2000;356:1327-8.

29. Arcalyst ${ }^{\circledR}$ (Rilonacept) [packet insert]. Tarrytown, NY. Regeneron Pharmaceuticals, Inc.; 2010.

30. Ilaris ${ }^{\circledR}$ (Canakinumab) [packet insert]. East Hanover, NJ. Novartis Pharmaceuticals Corporation; 2009.

31. Simponi ${ }^{\circledR}$ (Golimumab) [packet insert]. Horsham, PA. Centocor Ortho Biotech Inc.; 2010.

32. Stelara ${ }^{\circledR}$ (Ustekinumab) [packet insert]. Horsham, PA. Centocor Ortho Biotech Inc.; 2010.

33. Actemra ${ }^{\circledR}$ (Tocilizumab) [packet insert]. South San Francisco, CA. Genentech, Inc.; 2010.

34. Denlinger CS, Blanchard R, Xu L, Bernaards C, Litwin S, Spittle $\mathrm{C}$, et al. Pharmacokinetic analysis of irinotecan plus bevacizumab in patients with advanced solid tumors. Cancer Chemother Pharmacol. 2009;65(1):97-105.

35. European Medicine Agency. Clinical efficacy and safety guidelines introduction. London, UK. http://www.emea.europa.eu/ htms/human/humanguidelines/efficacy.htm. Accessed 04 Jan 2011.

36. Huang S-M, Temple R. Is this the drug or dose for you?Impact of ethnic factors in global drug development, regulatory review and clinical practice. Clin Pharmacol Ther. 2008;84(3):287-94. 
37. US Food and Drug Administration. Clinical Pharmacology Guidance for Industry. Rockville, MD. http://www.fda.gov/ Drugs/GuidanceComplianceRegulatoryInformation/Guidances/ ucm064982.htm. Accessed July 5, 2010.

38. Giacomini KM, Huang S-M, Tweedie DJ, Benet LZ, Brouwer KLR, Chu X, et al. The International Transporter Consortium, ITC. Membrane Transporters in Drug Development. Nat Rev Drug Discov. 2010;9:215-36.

39. US Food and Drug Administration. Briefing Information for the March 17, 2010 Meeting of the Pharmaceutical Science and Clinical Pharmacology Advisory Committee. Rockville, MD. 2010. http:// www.fda.gov/AdvisoryCommittees/CommitteesMeetingMaterials/
Drugs/AdvisoryCommitteeforPharmaceuticalScienceandClinical Pharmacology/ucm204260.htm. Accessed 5 July 2010.

40. Zhao P, Zhang L, Grillo J, Liu Q, Bullock JM, Moon YJ, et al. Application of physiologically based pharmacokinetic (PBPK) modeling and simulation in regulatory review. Clin Pharmacol Ther. 2011;89(2):259-67.

41. Huang S-M, Woodcock J. Commentary on ITC Membrane Transporters in Drug Development; Report from the FDA Critical Path Initiative Sponsored Workshop. Nat Rev Drug Discov. 2010;9:175-6.

42. Remicade ${ }^{\circledR}$ (Infliximab) [packet insert]. Horsham, PA. Centocor Ortho Biotech Inc.; 2010. 